\title{
Clasificar lo inclasificable: izquierda y derecha como categorías nativas
}

\author{
Ana Teresa Martinez
}

INDES-Universidad Nacional de Santiago del Estero / CONICET

$\mathrm{E}^{\mathrm{n}}$ n el presente trabajo me propongo apenas un ejercicio reflexivo para recordar algunas cuestiones teóricas que distan de ser novedosas, pero que vale la pena hacer presentes en este debate: qué significa decir que la categorización que nos ocupa es "nativa" del lenguaje político, pero buscamos explotar sus dimensiones analíticas. Vamos a discutir especialmente con el clásico trabajo de Bobbio Derecha e izquierda, ${ }^{1}$ recortando (a contraluz del texto y de un caso que construimos como cantera de ejemplos) la reflexión sobre las especificidades de la conceptualización de pretensión analítica que este autor plantea y la que puede atribuirse a lo que los antropólogos llaman una categoría "nativa", es decir, recogida de un lenguaje y un contexto social y cultural específicos.

Para no pensar en abstracto, vamos a plantear el problema a partir de los ejemplos de dos intelectuales de Santiago del Estero (Argentina) en el año 1943. Importa tener en cuenta que el material de archivo que utilizo no es homólogo para los dos casos: contamos con correspondencia privada, la totalidad de sus publicaciones y algunas actuaciones públicas de Bernardo Canal Feijóo. De Amalio

\footnotetext{
${ }^{1}$ Norberto Bobbio, Derecha e izquierda. Razones y significado de una distinción política, Madrid, Taurus, 1998.
}

Olmos Castro tenemos reiteradas actuaciones públicas relatadas por los diarios La Hora y El Liberal, así como las publicaciones que conocemos bajo su firma. No hemos encontrado situaciones públicas -ni privadas- en que Amalio Olmos Castro y Bernardo Canal Feijóo se crucen claramente durante este año, pero sí aparecen tomas de posición públicas y privadas de cada uno frente a acontecimientos similares. Escogemos a estos dos personajes para articular la reflexión por lo que tienen en común y de diferente: dos funcionarios públicos (de distinto carácter) e intelectuales que publicaban dentro y fuera de la provincia, preocupados por las problemáticas sociales. Elegimos el momento por su carácter de bisagra en la vida política nacional, un momento en que las posiciones se definen y distribuyen pero no son aún lo que llegarán a ser cuando la emergencia clara del peronismo divida aguas: las interpretaciones posibles sobre las tomas de posición están llenas aún de zonas grises y de potencialidades prácticas indefinidas.

En 1943 Bernardo Canal Feijóo² tenía 46 años, era abogado del Banco Hipotecario Na-

\footnotetext{
${ }^{2}$ Para más información sobre Bernardo Canal Feijóo cf. Ana T. Martinez, Constanza Taboada y Alejandro Auat, Los hermanos Wagner: entre ciencia, mito y poesía. Arqueología, campo arqueológico nacional y construcción de identidad en Santiago del Estero. 1920-1940, Bernal,
} 
cional y un escritor reconocido en el ámbito porteño, ganador de un premio nacional de cultura, colaborador de la revista Sur, autor de varios libros de poesía de vanguardia y de ensayos sobre cultura santiagueña, animador de la Asociación cultural La Brasa, promotor de investigaciones arqueológicas y de visitas culturales y científicas a su provincia, unido epistolarmente a una notable red de intelectuales y artistas de la región y del litoral. Vivía en Santiago del Estero y no había publicado aún sus principales ensayos, aunque trabajaba ya sobre Alberdi y reflexionaba sobre la configuración del territorio nacional.

Amalio Olmos Castro, ${ }^{3}$ catamarqueño radicado en Santiago del Estero al menos desde 1923, cuando lo encontramos como funcionario del yrigoyenista Maradona. En 1943, a los 58 años, era autor de artículos misceláneos publicados en periódicos y de numerosos informes estadísticos y trabajos de investigación social que daban cuenta de un conocimiento amplio de la provincia y sus problemas, abarcando desde la salud pública a la distribución de la propiedad de la tierra, proponiendo desde la cría del gusano de seda a la legislación del trabajo de empleadas domésticas. Eran publicados en revistas de circulación nacional pero específica, tales como $L a G a$ zeta algodonera o Maderil, en el Anuario Estadístico del Departamento Provincial del Trabajo de Santiago del Estero y El Boletín

\footnotetext{
Universidad Nacional de Quilmes, 2011, y Ana Teresa Martinez, "Bernardo Canal Feijóo, del problema del interior al interior como problema", en Carlos Altamirano y Adrian Gorelik (eds.), La Argentina como problema, Buenos Aires, Siglo XXI, 2018.

${ }^{3}$ Para más información sobre Olmos Castro cf. Ana T. Martinez, "Obrajes, leyes del trabajo y prácticas políticas. Las luchas por la construcción del Estado en el protoperonismo. Santiago del Estero (1943-1945)", Revista Andina, $\mathrm{n}^{\circ}$ 44, 2007, pp 116-141 y Ana T. Martinez, "Amalio Olmos Castro y la cuestión social en Santiago del Estero. El Departamento Provincial del Trabajo entre límites estructurales y conflictos ideológicos", Historia Regional, ${ }^{\circ}$ 40, 2019, pp 1-17.
}

del Departamento del Trabajo de Tucumán, así como en la revista del Departamento $\mathrm{Na}$ cional del Trabajo. Desde su creación en 1935 era director del Departamento Provincial de Estadísticas, Registro Civil y Trabajo de Santiago. En 1943 fue nombrado presidente de la recién creada Junta Honoraria de Investigaciones Sociológicas, dependiente de la Universidad Nacional de Tucumán (UNT), integrada además por Orestes di Lullo y Juan D. Chazarreta, vinculados de diversos modos al nacionalismo y a la militancia católica. También fue miembro correspondiente de la Comisión de Seguridad Social de la Universidad Nacional de La Plata, fundada por el socialista Alfredo Palacios durante su rectorado. Sin embargo, Olmos Castro no aparece integrado sino fugazmente en los proyectos colectivos de la intelectualidad local, predominantemente liberal (conservadora, radical o socialista) y nucleada en 1943 en el Colegio Libre de Estudios Superiores (CLES) y la Asociación Cultural La Brasa. ${ }^{4}$ Cuando se aparta de cuestiones estrictamente técnicas, la raíz social-católica de sus preocupaciones se deja traslucir en sus discursos, folletos y libros, tanto por su contenido como por las referencias en las que se apoya.

Si quisiéramos utilizar las categorías de $i z$ quierda o derecha como organizadoras de sus tomas de posición, públicas o privadas, objetivadas en textos o en acciones, durante 1943, nos veríamos en las dificultades que se pue-

\footnotetext{
${ }^{4}$ Orestes Di Lullo, de posiciones similares a Olmos Castro, es firmante del manifiesto de La Brasa y permanece vinculado hasta 1930. Olmos Castro nunca aparece relacionado con este grupo, hasta donde sabemos. En la convocatoria del PINOA (Primer Congreso de Planificación Regional del Noroeste Argentino, realizado en 1946) figura su nombre, pero luego desaparece de los boletines y del evento. En una sugerente carta de agosto de 1941, de Canal Feijóo a Figueroa Román (del Departamento de Sociografía de la UNT), aquel trata de asegurarse la intermediación entre este, Di Lullo y Olmos Castro, al tiempo que le propone que para trabajos sociológicos se vincule con el CLES recién creado.
} 
den prever recorriendo las caracterizaciones mencionadas y conociendo someramente el contexto. Lo primero a aclarar es que ninguno de los dos se autoclasifica como de izquierda o derecha, y tampoco utilizan estas categorías para clasificar a otros. Este es el primer punto: en este caso no recogemos la oposición como una categoría nativa de un espacio/tiempo social; es el investigador quien las introduce para una clasificación de pretensión analítica, pero, como es inevitable, extrayéndola de otros contextos donde funciona como nativa. Las primeras preguntas a hacerse son ¿qué puede aportar al análisis?, ¿qué nos permite hacer esta operación conceptual y cuáles son sus límites? En principio -podríamos decirofrece la comodidad de una organización quiasmática del campo de actores en un momento dado, que facilitaría una clasificación al menos aproximada de tomas de posición y acciones para delinear configuraciones de sentido. Esas tomas de posición y acciones deberían poder organizarse en torno a algún principio que permitiera clasificarlas a "la izquierda" o a "la derecha".

En su libro, Bobbio intenta delimitar algún núcleo de sentido que funcione como principio de clasificación. Partamos de sus puntos de llegada en el capítulo séptimo: luego de haber precisado desde el inicio que dejará de lado el significado "emocional" para centrarse en el "descriptivo" y de considerar que izquierda y derecha son "términos" "exclusivos" y "exhaustivos", luego de barrer y discutir con trabajos anteriores que intentaban -en la década de 1990- dar de baja la oposición o recuperarla, Bobbio fundamenta la pertinencia de las categorías izquierda/derecha en el uso que continúa haciéndose de ellas en el campo político. Según afirma:

los términos "derecha" e "izquierda" siguen estando vigentes en el lenguaje político. Todos aquellos que los utilizan no dan en absoluto la impresión de usar palabras en balde porque se entienden muy bien entre sî".

Es decir, en el discurso político de la época continuarían activas porque permitirían que los interlocutores comprendieran aquello de lo que se está hablando. Aceptado este punto de partida pragmático, trata de entender su funcionamiento y define dos criterios a combinar para precisar la categorización: igualdad/desigualdad como criterio central, libertad/autoridad como criterio secundario.

Volviendo a los discursos y las acciones de nuestros personajes, apliquemos los criterios de Bobbio en ese complejo año argentino de 1943. Respecto del criterio secundario de la libertad: 1) Aunque manifiesta no poder expresarse públicamente por su propia condición de funcionario público, Canal Feijóo escribe a Figueroa Román, el sociólogo tucumano, para solidarizarse con él cuando es separado de su cargo de juez; hace lo mismo con Francisco y José Luis Romero, así como con Alfredo Orgaz cuando son expulsados de sus cátedras; apoya epistolarmente a Eugenio Pucciarelli en sus preocupaciones por la libertad de cátedra en la UNT. 2) Por el contrario, Olmos Castro no solo no defiende desde su cargo a los dirigentes gremiales, integrantes del Consejo del Trabajo, que son encarcelados por razones ideológicas en mayo de ese año, sino que cuando sus bases los vuelven a elegir meses después para que vuelvan a ocupar sus puestos, ya consumado el golpe de Estado, el director del Departamento del Trabajo induce desde su cargo la formación de una nueva Federación gremial para integrar el Consejo con dirigentes alejados de "ideologías extrañas". Su ideología católica parece prevalecer en la base de un anticomunismo menos preocupado por las libertades políticas individuales. No se trata de los mismos acontecimientos en uno y

\footnotetext{
${ }^{5}$ Norberto Bobbio, Derecha e izquierda, p. 89.
} 
otro caso, pero si los tratamos con los instrumentos clasificatorios de Bobbio se vuelven comparables. Según los criterios enunciados, deberíamos decir que en estas tomas de posición Canal Feijóo está a la izquierda respecto de Olmos Castro por su defensa de la igualdad ante la ley y la libertad política.

Sin embargo, vayamos al criterio principal, el de la igualdad: la clasificación se matiza y hasta podría invertirse si tenemos en cuenta las tomas de posición pública en ese mismo año de ambos funcionarios respecto al obraje maderero, un tema particularmente sensible en Santiago del Estero. 1) Ya desde la sequía de 1937 Canal Feijóo había tomado posición responsabilizando a los obrajes de las consecuencias de la deforestación sobre el clima, como una de las causas de la sequía. Sin embargo, cuando se refiere a las consecuencias sociales de ese modelo de producción forestal, se preocupa por la migración interna, por el despoblamiento de las zonas rurales y el abandono de la pequeña producción rural, por la pérdida de referencias culturales de las clases populares tras la desaparición del bosque, pero poco dice sobre las dramáticas condiciones de vida de los hacheros. 2) Olmos Castro, al contrario, en su libro El trabajo -y en los informes que publica fundamentando multas a los obrajeros por incumplimiento de leyes básicas- describe minuciosamente la resistencia de los propietarios a hacerse cargo de accidentes de trabajo, los precios de las proveedurías de propiedad obrajera, el pago en vales y no en moneda nacional, el trabajo a destajo, la falta de agua potable, en suma, condiciones laborales que califica como de semi-esclavitud.

Si bien Canal Feijóo algunos años después definió el obraje como "catástrofe social”, no está claro en sus escritos cuánto contaba allí la explotación obrera y cuánto los otros problemas más estructurales y culturales. La confrontación de Olmos Castro con los obrajeros cobró en la segunda mitad de 1943 características épicas, por sus inspecciones sor- presivas, sus denuncias administrativas $y$ judiciales a obrajeros y sus enfrentamientos con el prestigioso diario El Liberal, que defendía la llamada "industria madre" y descalificaba al director del Departamento Provincial de Trabajo por no contar con título en leyes. No hemos encontrado, ni en la correspondencia ni en artículos periodísticos o libros de Canal Feijóo, alusión alguna al conflicto ni a esta dimensión del mundo del obraje. Este silencio, sumado a los indicios anteriores, pueden sugerir una posición que, en términos de Bobbio, estaría a la derecha de Olmos Castro cuando se trata de desigualdad económica y social, del ejercicio efectivo de la libertad y los derechos para esas clases populares que analizaba y ponderaba desde el punto de vista de la creatividad cultural.

Volvemos entonces a la pregunta anterior: ¿qué nos aporta la clasificación si nos proponemos usarla como categoría analítica? Bobbio sostiene que se trata de "términos" "exclusivos" y "exhaustivos", es decir, al centrarse en el significado "descriptivo" decide aplicarles la lógica proposicional y el principio de no contradicción. Sin embargo, el carácter relacional que define la "izquierda" y la "derecha" abre a mil matices y desplazamientos semánticos según el actor, el caso y el principio de clasificación que se priorice. El planteo se sostiene en aquella perspectiva solo si además explicitamos otros aspectos que Bobbio aclara: el uso de estos principios de izquierda y derecha supone el marco de las sociedades políticas ilustradas, con principio de mayorías y sistema político democrático. Podríamos preguntarnos por qué. Supone entonces también -aunque no lo explicita- sociedades "modernas" con diferenciación institucional y cultural de campos (Bourdieu) y esferas (Weber). ${ }^{6}$

\footnotetext{
${ }^{6}$ No podemos extendernos aquí, pero mientras "campos" refieren a una organización social, que pone el acento en la producción de un determinado tipo de actores y de cosas específicas en juego, "esferas" refiere a un
} 
En este marco, la no contradicción puede salvarse solo prestando atención a los "respectos" (respecto de qué se dice lo que se dice) de los enunciados. Volviendo a nuestros ejemplos, si ampliáramos los "respectos" y analizáramos también las posiciones sobre el rol de las mujeres en la sociedad -explicitadas por uno y otro de nuestros personajes en correspondencia y en publicaciones- podríamos afirmar también que Canal Feijóo es de izquierda en términos culturales y de centro-derecha en términos políticos y sociales, mientras que Olmos Castro se situaría en el centro-izquierda respecto de cuestiones sociales y de derecha en las políticas y culturales. ¿Quién está más globalmente a la derecha y quién a la izquierda entonces? Si el criterio principal es el de la igualdad, deberíamos inclinarnos por Olmos Castro, atravesado por la contradicción de preocuparse por la sindicalización de las trabajadoras domésticas pero para devolverlas lo antes posible al ámbito de sus propios hogares y familias. El problema parece estar precisamente en separar lo que en las disposiciones de los sujetos y las tomas de posición es más bien inseparable.

Al tocar más de lleno el tema de la lógica de las clasificaciones, parecemos llegar a un punto que Bobbio no vio, o no vio suficientemente. ¿En qué medida es posible separar el sentido "descriptivo" de nuestra clasificación para poder utilizarlo sin equívocos como categoría analítica? ¿No se confunde en el análisis de Bobbio la categoría analítica con la categoría nativa? ¿Y qué es entonces una “categoría nativa"? Concediendo el encuadre en las sociedades modernas hijas de la ilustra-

aspecto de los campos: el suelo de creencias, reglas y valoraciones que suele ser concomitante a cada espacio social diferenciado. Dicho a la inversa, las esferas de valor refieren a una diversificación de modos de valorar y de reglas de funcionamiento, la noción de campo desarrolla las condiciones sociales de producción de lo que menciona Weber. ción: ¿nativa de qué tipo de espacio social (a la vez campo y esfera de valor) sería esta categorización? En principio parece obvio que para Bobbio es nativa del mundo político. Sin embargo, sabemos que la categorización tiene una prehistoria que -si lo miramos desde el punto de vista antropológico- no puede sino pervivir en la categorización política.

Como es sabido, izquierda/derecha forma parte de las oposiciones que podemos remontar, si nos remitimos a textos conocidos de la historia europea, hasta los presocráticos, particularmente los pitagóricos. Encontramos en esa tradición explicitadas las oposiciones derecha/izquierda, macho/hembra, reposo/movimiento, unidad/pluralidad, rectilíneo/curvo, luz/tinieblas, bien/mal, donde los términos opuestos quedan asociados no solo horizontal sino verticalmente: la derecha es el macho, el reposo, la unidad, lo rectilíneo, la luz, el bien. En esta mitología situada en la prehistoria de la filosofía griega, la izquierda es la hembra, el movimiento, la pluralidad, lo curvo, las tinieblas, el mal. Sin embargo, estas oposiciones han sido encontradas, con asociaciones verticales similares, en múltiples sociedades antiguas y contemporáneas, expresadas en rituales, textos y prácticas, especialmente -pero no solo- en torno al Mediterráneo.

Para la antropología, el que nos preocupa no sería sino un caso particular de los ejes organizadores que produce el cuerpo como operador analógico: arriba/abajo, izquierda/derecha, delante/detrás, dentro/fuera son las categorías que organizan nuestra relación con el espacio a partir de nuestro cuerpo. Y como el cuerpo carga las disposiciones que se producen en la socialización, estas relaciones espaciales se van connotando socialmente mientras en las relaciones sociales se va instituyendo, naturalizando, valorando e incorporando a la vez prácticas y disposiciones de los sujetos y las instituciones. Los ejemplos en el lenguaje, gestos y textos que sostienen las creencias sobre la vida, la muerte y el sentido (que las sociedades occi- 
dentales sitúan en un ámbito particular que 1lamamos religioso) son innumerables. Pero todas estas oposiciones primitivas (en el sentido de fundamentales) también configuran mucho del lenguaje, gestos, textos e instituciones de ese otro espacio de la creencia y la acción que es el campo/esfera política. Y el famoso caso de la Asamblea Francesa post-revolucionaria que sirve con frecuencia de referencia a los análisis historiográficos, no sería, mirado desde aquí, sino un caso particular -al menos inicialmente- de esta experiencia.

Dicho esto, vale preguntarse cómo funciona esta mitología -que, como nos enseñaba Wittgenstein, carga el lenguaje y organiza mundos $-^{7}$ en el campo de la política, y qué implicaciones epistemológicas tiene ese funcionamiento. Por aquí nos acercamos a un aspecto que Bobbio no considera: izquierdal derecha nunca pueden ser completamente exclusivos y exhaustivos, como surge de nuestros ejemplos y de muchos otros que pudiéramos invocar. Y esto, precisamente por tratarse de una categoría nativa. Su potencial descriptivo es clasificatorio, pero al modo de una categoría de la práctica, más coherente de lo que se espera, pero menos de lo que se pretende. El campo político, aun en el juego democrático de las sociedades modernas, no es solo un espacio de creencias, es sobre todo un espacio de valoraciones (de las que no se puede excluir sus dimensiones emocionales), de intereses y de acciones, y lo que allí se dice no está orientado a la contemplación, a la disección científica en orden al puro conocimiento, sino a la práctica, a producir efectos en el mundo, cambiar juegos de fuerzas y relaciones de poder. Como todo lenguaje de la prác-

\footnotetext{
${ }^{7}$ Los párrafos que siguen están especialmente inspirados en las reflexiones de Ludwig Wittgenstein, Observaciones sobre la Rama Dorada de Frazer [1967], Madrid, Tecnos, 1992 y Pierre Bourdieu, Le sens pratique, París, Minuit, 1980 y Curso de sociología general [2015], Buenos Aires, Siglo XXI, 2019.
}

tica, está profundamente anclado en el cuerpo y funciona, no con el lenguaje unívoco y la lógica proposicional sino mediante operaciones analógicas, posibles porque aprovechan la ductilidad del lenguaje ordinario. Bobbio tiene razón: la dupla persiste, y esa persistencia de hecho es suficiente criterio para aceptar que de algún modo funciona. Pero entiendo que no funciona como él cree, sino que persiste porque tiene la ductilidad lógica de los juegos analógicos del lenguaje natural, pleno de deslices semánticos. Y este no es su defecto, sino su condición de uso, de significatividad. La política es mundo de práctica y su coherencia es la de la acción. Allí los discursos son acciones y si valen como tales, es porque el cambio constante de "respectos" es su condición de posibilidad.

Es una lógica analógica la que posibilita los desplazamientos. La analogía es el "demonio" que la hace funcionar y, como en la hipótesis de Descartes del genio maligno, construye mundos de sentido. Entendemos de qué hablamos, pero no pidamos a las palabras que expliquen exhaustivamente nada por su significado aislado, definidas como en el diccionario. Izquierda y derecha son tales respecto de ciertos sujetos en un campo, pero además solo son útiles moviéndose de un plano al otro en un juego de referencias múltiples. Pretender estabilizarlas y darles un sentido preciso como categorías analíticas -como en el debate en que se enredaban los cientistas políticos en tiempos de Bobbioes confundir ambos tipos de categorías y cambiar de naturaleza los discursos: entre el discurso político donde estos principios de clasificación funcionan como pez en el agua y el discurso del cientista político o del historiador hay una diferencia epistemológica que nos debemos recordar.

Como dice también Bobbio, con razón, hoy el carácter positivo o negativo adjudicado a "izquierda" o "derecha" en el campo político es objeto de luchas, y la serie de asociaciones 
pitagóricas que evocábamos antes ya no es asumida como formando parte de un sentido común que va de suyo. Podemos aventurar, siguiendo el hilo del razonamiento, que esto es así porque no hay continuidad en las reglas de juego entre unos espacios sociales y otros. Y en esa diversidad de lógicas, el lenguaje natural, que es de común patrimonio, es objeto de modificaciones por sus diversos modos de uso: esfuerzos de definición y formalización en las disciplinas de las ciencias sociales; potenciación de sus dimensiones expresivas y simbólicas en el espacio del arte; preponderancia de las dimensiones performativas en política, donde sobre todo decir es hacer, clasificar es acusar, describir es incidir. Y los agentes sociales nos movemos deslizándonos y jugando siempre en varios tableros a la vez, ignorando límites para optimizar nuestras posibilidades. Pero al proponer un análisis de pretensiones científicas es importante recordarlo.

En suma, solo queríamos traer a la reflexión, valiéndonos de algunos ejemplos, que izquierda/derecha son útiles como organizadores de un discurso del sentido práctico, pero son limitadas y riesgosas cuando las transponemos como categorías de un discurso de pretensiones científicas que, como el de las ciencias sociales, no puede recurrir a un lenguaje enteramente formalizado, sino que se mueve en ese medio del lenguaje natural, con categorías que siempre son nativas de algún lugar y tiempo. Las categorías clasificatorias de la práctica política son útiles, pero difíciles de transponer precisamente por su enorme complejidad significativa cuando están "en su salsa", es decir, en el uso político.

\section{Bibliografía citada}

Bobbio, Norberto, Derecha e izquierda. Razones y significado de una distinción política [1995], Madrid, Taurus, 1998.

Bourdieu, Pierre, Le sens pratique, París, Minuit, 1980. Curso de sociología general [2015], Buenos Aires, Siglo XXI, 2019

Martínez, Ana Teresa, Taboada, C y Auat, L. A., Los hermanos Wagner: entre ciencia, mito y poesía. Arqueología, campo arqueológico nacional y construcción de identidad en Santiago del Estero. 1920-1940, Bernal, UNQ, 2011.

Martinez, Ana Teresa, "Bernardo Canal Feijóo, del problema del interior al interior como problema", en C. Altamirano y A. Gorelik (comps.), La Argentina como problema, Buenos Aires, Siglo XXI, 2018, pp. 99-112.

“Obrajes, leyes del trabajo y prácticas políticas. Las luchas por la construcción del Estado en el protoperonismo. Santiago del Estero (1943-1945)", Revista Andina, $\mathrm{n}^{\circ}$ 44, 2007, pp. 116-141.

"Amalio Olmos Castro y la cuestión social en Santiago del Estero. El Departamento Provincial del Trabajo entre límites estructurales y conflictos ideológicos", Historia Regional, $\mathrm{n}^{\circ}$ 40, 2019, pp. 1-17.

Wittgenstein, Ludwig, Observaciones sobre la Rama Dorada de Frazer [1967], Madrid, Tecnos, 1992. 


\section{Resumen/Abstract}

\section{Clasificar lo inclasificable: izquierda y derecha como categorías nativas}

El trabajo consiste en un ejercicio reflexivo sobre cuestiones teóricas acerca del significado de "izquierda" y "derecha" como categorías "nativas" del lenguaje político. Discutimos con el clásico trabajo de Bobbio Derecha e izquierda, recortando conceptos a contraluz del texto y de un caso que construimos como cantera de ejemplos: la confrontación de actuaciones y discursos públicos de dos intelectuales de Santiago del Estero (Argentina) en el interregno entre el golpe de Estado de junio de 1943 y la asunción del primer gobierno peronista en 1946. Se advierte cómo las categorías clasificatorias de la práctica política son útiles en ese campo, pero difíciles de transponer al debate científico precisamente por su enorme complejidad significativa cuando están "en su salsa", es decir, en el uso político.

Palabras clave: Categorías nativas - Campo político - Lenguaje natural - Oposiciones binarias
To classify the unclassifiable: Left and right wing ideas as native categories

The work consists of a reflective exercise on theoretical questions about the meaning of "left" and "right" as "native" categories of political language. We discuss Bobbio's classic work "Right and left", cutting back concepts from the text and a case that we consider to be a quarry of examples: the confrontation of actions and public speeches of two intellectuals from Santiago del Estero (Argentina) between the coup d'etat in June, 1943 and the inauguration of the first Peronist government in 1946. It is noted that the classificatory categories of political practice are useful in this field, but also difficult to transpose into scientific debate due to their enormous significant complexity in political use.

Keywords: Native categories - Political field Natural language - Binary oppositions 